\title{
Las controversias y los actuales desafíos en la patentabilidad de los biorrecursos antárticos ${ }^{1}$
}

\section{Controversies and present challenges of patenting Antarctic bio-resources}

\author{
Catalina Sepúlveda Illanes \\ catalina.sepulveda.i@ug.uchile.cl \\ Egresada de Derecho, Universidad de Chile.
}

Resumen: La bioprospección es una actividad científica relevante en la Antártica, pero también de importancia económicas. Sin embargo, desde la perspectiva del Sistema del Tratado Antártico y del uso de los recursos antárticos existen una serie de cuestiones no resultas. Una de ellas es la referida a la patentabilidad de los bio-recursos antárticos.

Palabras claves: Bioprospección - Antártica - Sistema del Tratado Antártico - patentabilidad

Abstract: Bioprospecting is a relevant scientific activity in Antarctica but it is also an economical important issue. However, from the Antarctic Treaty System point of view and the Antarctic resources exploitation there are several issues not resolved. One of this matter is the patenting of Antarctic bio-resources.

Keywords: Bioprospecting - Antarctica - Antarctic Treaty System - Patenting

\section{Introducción}

La sociedad moderna se ha caracterizado por el rol protagónico otorgado al conocimiento y a la información, surgiendo la necesidad de favorecer la capacidad creadora de los individuos mediante la protección de sus invenciones. Aquello se ha logrado a través del derecho de propiedad intelectual, particularmente mediante el establecimiento de patentes, las cuales han jugado un importante papel en las invenciones basadas en los biorrecursos antárticos. No

1 Ponencia presentada en las Jornadas Chilenas de Derecho Antártico 2019, Facultad de Derecho, Universidad de Chile, 28 de marzo del 2019. 
Las controversias y los actuales desafíos en la patentabilidad de los biorrecursos antárticos Catalina Sepúlveda Illanes

obstante, actualmente no existe una regulación especial sobre la materia, concurriendo al mismo tiempo el derecho de patentes y el Sistema del Tratado Antártico. Frente a ello, debemos preguntarnos cómo confluyen ambas regulaciones, cuáles son los conflictos suscitados y qué desafíos presentan actualmente para la comunidad internacional.

\section{La bioprospección}

En principio, debemos adelantar que no existe una definición uniforme sobre la bioprospección, la cual varía según los actores involucrados y a su contexto. No obstante, todas las definiciones coinciden en que es la actividad de exploración científica dedicada a buscar algo útil en la naturaleza, cuyo fin último es la comercialización del producto final.

En nuestro caso se traduce en la búsqueda de material biológico y genético antártico, el cual tienen un potencial valor comercial, debido a sus componentes únicos para el desarrollo de algún producto. Es respecto a este último que probablemente se registre una patente para ejercer el dominio exclusivo de su explotación.

En la Antártica, estas investigaciones se habrían iniciado a partir la década de los sesenta del siglo pasado, las que han aumentado considerablemente debido a los avances en la industria biotecnológica. Aquello lo podemos corroborar con las últimas cifras otorgadas públicamente, las cuales fueron hechas por un estudio del Instituto de Estudios Avanzados de la Universidad de las Naciones Unidas, "según la cual, entre 1988 y 1989 existía una sola patente de invención procedente de la bioprospección antártica, mientras que para enero del año 2013 la página web www.bioprospector.org registra 185 patentes" (Villamizar, 2013: 57).

Dentro de los principales usuarios de los recursos antárticos está la industria farmacéutica y de la tecnología médica (23\%), seguida de la industria de alimentos y bebidas (20\%) y la biología molecular y la biotecnología $(18 \%)^{2}$.

${ }^{2}$ XXXI Reunión Consultiva del Tratado Antártico (Kiev, 2008), WP 11 (Bélgica) 7.

Número de página no utilizable para citar

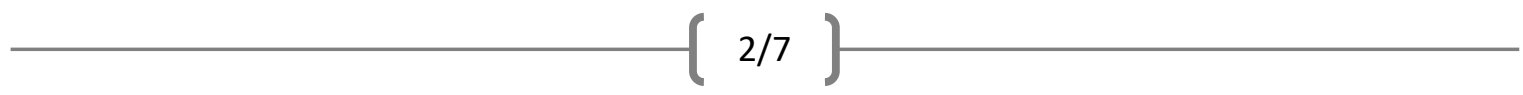




\section{La patentabilidad de los biorrecursos antárticos}

Cabe señalar, que los proyectos de bioprospección en la Antártica generalmente conllevan un alto costo debido al medio hostil y lejano, por lo que el inversionista debe tener como mínimo, la posibilidad de obtener una recompensa material. Por esta razón, generalmente se constituyen patentes, ya que garantizan un "derecho exclusivo sobre una invención, el producto o proceso que constituye una nueva manera de hacer algo, o propone una nueva solución técnica a un problema" (OMPI: 5). Como contrapartida, en algún momento el titular de la patente deberá divulgar la información técnica relativa a la invención para que pase a ser parte del dominio público.

En las patentes, el elemento esencial es la invención, en contraposición con los meros descubrimientos. En consecuencia, se podría patentar un producto basado en material biológico o genético antártico en tanto posea características notoriamente diversas a las que le pertenecen naturalmente.

En razón del elemento creador humano de las patentes, es porque se otorga el monopolio de su explotación por un tiempo determinado, la regla general es de 20 años máximo. Posterior a ello, surge la obligación de divulgación con el objeto de que "terceros puedan beneficiarse de los nuevos conocimientos y contribuir así al desarrollo tecnológico" (INAPI).

Por otra parte, para mantener el monopolio de explotación por el lapso otorgado, los privados suelen pactar cláusulas de confidencialidad, para tener conocimiento y ejercicio exclusivo sobre las observaciones y resultados científicos obtenidos.

\section{Las controversias asociadas a la patentabilidad de biorrecursos antárticos}

El problema se produce principalmente por la naturaleza monopólica de las patentes, la que entra en conflicto con los principios fundamentales del Tratado Antártico: la libertad de la investigación científica y la cooperación internacional, consagrados en los artículos II y III respectivamente. Debido a la exclusividad intrínseca de las patentes y las cláusulas de confidencialidad, no existe disposición pública del conocimiento y la utilización de los resultados científicos obtenidos en razón del trabajo patentado, aún cuando son recursos que cabe discutir si deberían regirse por los principios del Sistema del Tratado Antártico. 
Por otra parte, también se ha planteado el potencial conflicto entre el derecho exclusivo del titular de la patente y la pregunta por la soberanía en la Antártica, en razón del artículo IV del Tratado Antártico. Al respecto, la Convención de Diversidad Biológica u otros instrumentos internacionales eventualmente aplicables, disponen que los Estados son soberanos sobre los recursos naturales. En razón de ello, están legitimados para efectuar acuerdos que dispongan de los mismos y por tanto, llevar a cabo la bioprospección. Por lo que algunos autores han planteado la duda sobre la legitimidad de la apropiación de los recursos antárticos mientras la soberanía esté en suspenso (Connolly-Stone, 2005: 79). No obstante, no sería correcta dicha apreciación, puesto que el artículo IV no suspende la soberanía, sino las controversias relacionadas a ella.

Sin perjuicio de lo anterior, en general las discusiones se han enfocado en el conflicto entre el derecho de patentes y los principios de libertad científica y cooperación internacional. Respecto al cual, la primera dificultad la encontramos en la fuente de las patentes, pues al ser un derecho otorgado por un Estado, su regulación queda sujeta a la legislación nacional y a la jurisprudencia de cada jurisdicción. Lo que se expresará específicamente respecto al alcance de la protección brindada a la patente y su respectiva interpretación, pudiendo ser diferente aún cuando se refiera a una invención biológica idéntica (Tvedt, 2010: 7). En consecuencia, el primer problema está en la ausencia de un sistema armónico en la regulación de la propiedad intelectual sobre los biorrecursos antárticos.

En cuanto a la accesibilidad pública del origen de la invención, se ve obstaculizada por el Tratado de Budapest sobre el Reconocimiento Internacional del Depósito de Microorganismos a los fines del Procedimiento en materia de Patentes (1977, en vigor 1980) que no regula la disponibilidad de los resultados de la investigación a otros que no sean el titular de la patente (Tvedt, 2010: 6). De manera que, en este caso la obligación de divulgación no podría realmente satisfacer el principio de libertad científica, pues su cumplimiento radica en la disponibilidad del gen aislado o el material patentado para otros investigadores.

En un mismo sentido, se ha discutido el conflicto entre la Convención de Diversidad Biológica (1992, en vigor 1993) y el Acuerdo sobre los Aspectos de los Derechos de Propiedad Intelectual relacionados con el Comercio (1994, en vigor 1995), respecto a la difusión sobre el origen del material biológico de las patentes. Lo anterior, debido a la posible patentabilidad de los microorganismos y los procedimientos no biológicos o microbiológicos para la producción de plantas y animales ${ }^{3}$. Aquello adquiere relevancia por la aplicación potencial de la Convención de Diversidad Biológica sobre los biorrecursos antárticos, sirviendo además por su regulación sobre el acceso de los recursos genéticos y la forma de distribución de los beneficios.

3 En conformidad del artículo 27, número 3, apartado (b) del Acuerdo sobre los Aspectos de los Derechos de Propiedad Intelectual relacionados con el Comercio.

Número de página no utilizable para citar 
Es así que, damos cuenta que este conflicto aparece de manera transversal en materia de los biorrecursos, principalmente por la demanda de los países en vías de desarrollo, los que requieren a los solicitantes de patentes la divulgación sobre la procedencia del material biológico. No obstante, no han obtenido una respuesta positiva por el gran rechazo de la industria y los países desarrollados (Tvedt, 2010: 6).

Por último, esta restricción al acceso del gen aislado u otra expresión biológica a otros investigadores, impide materializar los principios de investigación científica e incluso posibles relaciones cooperativas entre Estados. En la medida que, indirectamente el patentamiento limitaría la posibilidad de seguir investigando aquella planta o animal, pues este componente otorgará el mismo resultado ante diferentes procedimientos por su construcción genética natural.

\section{Desafíos vinculados a los biorrecursos antárticos}

En la actualidad, aún no existe determinación en cuanto a la regulación de la bioprospección antártica, siendo un tema pendiente desde la XXIII Reunión Consultiva del Tratado Antártico (Lima, 1999). Por consiguiente, la regulación de derechos de propiedad intelectual es la que se ha impuesto sobre los biorrecursos antárticos, fijando lo que está abiertamente disponible y lo que no (Tvedt, 2010: 9). Por tanto, es esperable que se contravenga el Sistema del Tratado Antártico, ya que el material biológico y genético queda sujeto al derecho de patentes de cada país, influenciado por factores político-económicos externos a los temas antárticos.

Entonces, cabe preguntarse cómo podemos compatibilizar la protección de los biorrecursos antárticos y al mismo tiempo, el sistema de patentes existente. La respuesta a esta pregunta debe provenir de la comunidad internacional, la que deberá resolver lo que podría ser un equilibrio político-jurídico ante esta incompatibilidad. Una posible solución sería a través de la ley de ponderación, siendo el primer paso la definición del grado de no satisfacción o de afectación de uno de los principios. Luego, definir la importancia de la satisfacción del principio que juega en sentido contrario. Finalmente, definir si la importancia de la satisfacción del principio contrario justifica la restricción o la no satisfacción del otro (Alexy, 2003: 13).

Sin perjuicio de lo anterior, no debemos olvidar que el marco de la deliberación debe realizarse en términos realistas, en el sentido que "las obligaciones del artículo III no se han interpretado históricamente como un requisito para que ningún científico entregue los datos o conocimientos que ha adquirido en cualquier área de investigación” (Hemmings, 2009, 12). Por lo que, la discusión debería estar dirigida a reducir los obstáculos que conlleva el derecho de patentes frente 
al Sistema del Tratado Antártico, y así establecer mecanismos que permitan en mayor medida la libertad de investigación científica e incentive la cooperación internacional.

Aún cuando en las Reuniones Consultivas del Tratado Antártico han existido intentos de discutir la regulación de la bioprospección, no se ha logrado una solución clara frente a las indefiniciones e incompatibilidades de la bioprospección antártica dentro del Sistema del Tratado Antártico. Por lo que pareciera que la mayoría de las partes del Tratado Antártico están satisfechas con el avance de las actividades de la bioprospección, en tanto se pueda evitar el conflicto de intereses entre los distintos actores. Sin embargo, no se puede desconocer el hecho de que cada vez se hace más insostenible la inmutabilidad de la comunidad internacional frente al aumento de la bioprospección antártica.

\section{Conclusiones}

En consideración a lo expuesto, podemos afirmar con propiedad que existen diversas dificultades relacionadas a la patentabilidad sobre los biorrecursos antárticos en conformidad al Sistema del Tratado Antártico. Principalmente, por la controversia entre el derecho de patentes y los principios de libertad científica y la cooperación internacional del Tratado Antártico.

Pues, en estos momentos al decidir entre la regulación de una u otra, no se logra entregar un marco jurídico completo e idóneo respecto a los biorrecursos antárticos. No obstante, este problema no es desconocido dentro de la comunidad antártica, sino que ha sido debatido en diversas Reuniones Consultivas del Tratado Antártico, pero continúan sin tener una determinación clara al respecto.

Por ello, surge la necesidad de encontrar un equilibrio político-jurídico ante esta incompatibilidad, para velar por la efectiva protección a los biorrecursos antárticos y evitar potenciales conflictos entre los Estados reclamantes y no reclamantes de soberanía en la Antártica. Por lo cual, la decisión necesariamente debe surgir dentro de las Reuniones Consultivas del Tratado Antártico y no dejar que la práctica y el derecho doméstico determinen este asunto. 


\section{Referencias bibliográficas}

AlEXY, Robert (2003). "La formula del peso". En: Carbonell, Miguel. (Ed.), Elprincipio de proporcionalidad y la interpretación constitucional. Ministerio de Justicia y Derechos Humanos, Quito.

Connolly-Stone, Kim (2005). "Patents, Property Rights and Benefit Sharing". Gateway Antartica Special Publication Series, Universidad de Canterbury, 5017.

Hemmings, Alan (2009). Biological Prospecting in the Antarctic Treaty Area. Reunión Intergubernamental de Expertos en Prospección Biológica en el área del Tratado Antártico, Baarn, 12.

https://www.researchgate.net/publication/256002759_Biological_Prospecting_in_the_Antarc tic_Treaty_Area [Consultado: 20.jul.2019].

INAPI (Instituto Nacional de Propiedad Industrial, Chile). Patente de invención. https://www.inapi.cl/patentes/tipos-de-patentes/invencion [Consultado: 20.jul.2019].

OMPI (Organización Mundial de Propiedad Intelectual), ¿Qué es la propiedad intelectual? https://www.wipo.int/edocs/pubdocs/es/intproperty/450/wipo_pub_450.pdf [Consultado: 20.jul.2019].

TVedt, Morten (2010). "Patent law and bioprospecting in Antarctica”. Polar Record.

Villamizar, Fernando (2013). "La Bioprospección Antártica: indefiniciones e incompatibilidades con el sistema del Tratado Antártico", International Law, Revista Colombiana de Derecho Internacional (23): 53-86. http://www.scielo.org.co/pdf/ilrdi/n23/n23a03.pdf [Consultado: 20.jul.2019]. 